\title{
RAPID QUENCH IN AN
}

\section{ELECTROSTATIC LEVITATOR}

Michael P. SanSoucie and Jan R. Rogers

NASA Marshall Space Flight Center

Douglas M. Matson

Tufts University 


\section{Outline}

- MSFC Electrostatic Levitation (ESL) Laboratory

- Rapid Quench System

- Motivation

- Quench Medium

- Quench Videos

- Future Work 


\section{The MSFC Electrostatic Levitation (ESL) Laboratory}

- The MSFC ESL Lab is a national resource for researchers developing advanced materials for new technologies

- Can process elements, alloys, refractory metals, superalloys, ceramics, oxides, and glasses

- The lab typically measures thermophysical properties

- Density

- Surface tension

- Viscosity

- $\quad$ Provides ground-based support for US investigators with levitation experiments on ISS

- ESA's Materials Science Laboratory Electromagnetic Levitator (MSL-EML)

- JAXA's Electrostatic Levitation Furnace (ELF)

- Recently upgraded with a rapid quench system

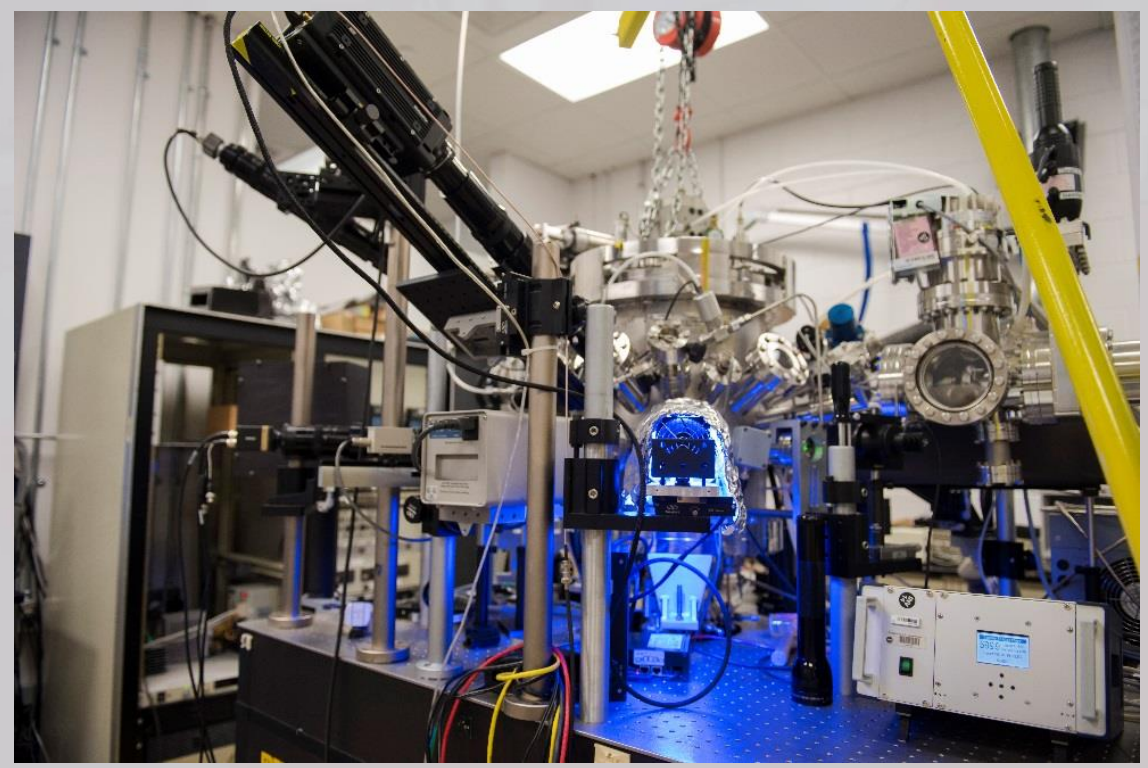




\section{Rapid Quench System}

- First submersion quench system inside an electrostatic levitator

- Allows samples to be dropped into a quench vessel that can be filled with a low melting point material, as a quench medium

- Thereby allowing rapid quenching of undercooled liquid metals

- Quench vessels can be raised or lowered using the same stem that is used to launch samples

- Up to 8 quench vessels can be loaded into the quench wheel

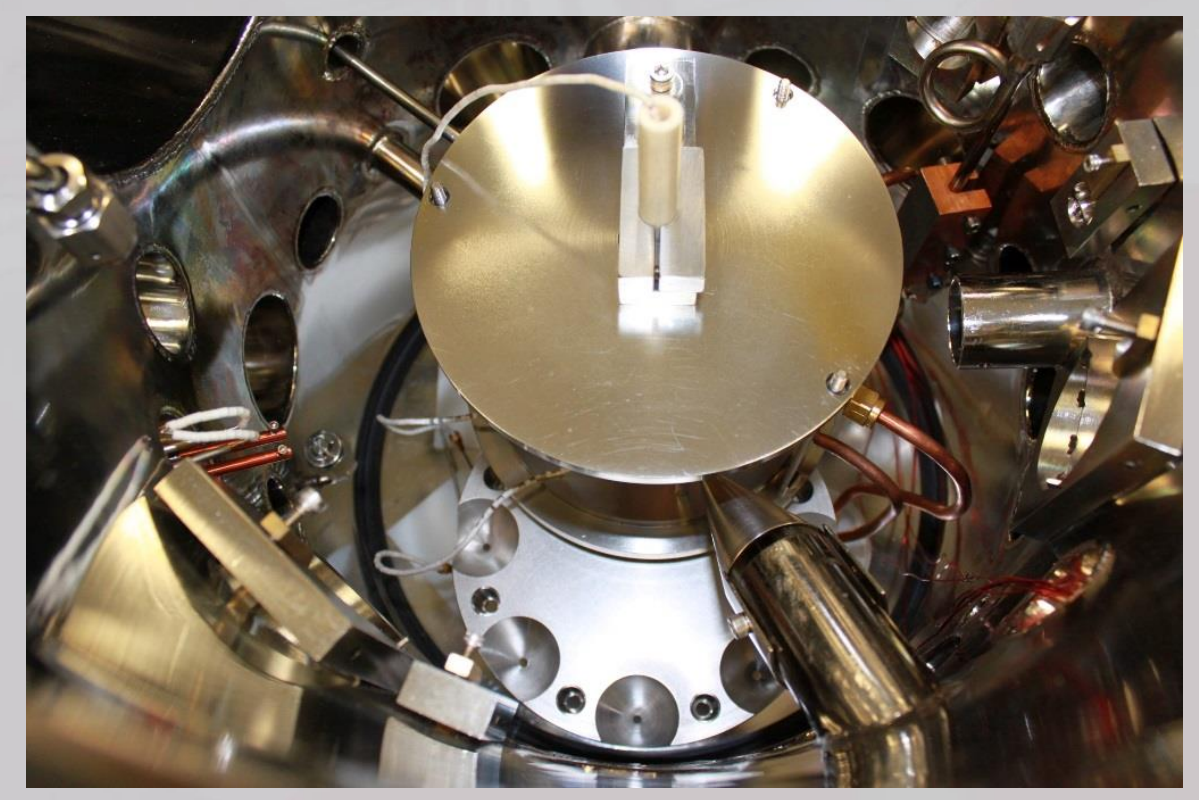

- Wheel is indexed with servo motors that are controlled with LabVIEW software 


\section{Exploded View of Rapid Quench System}

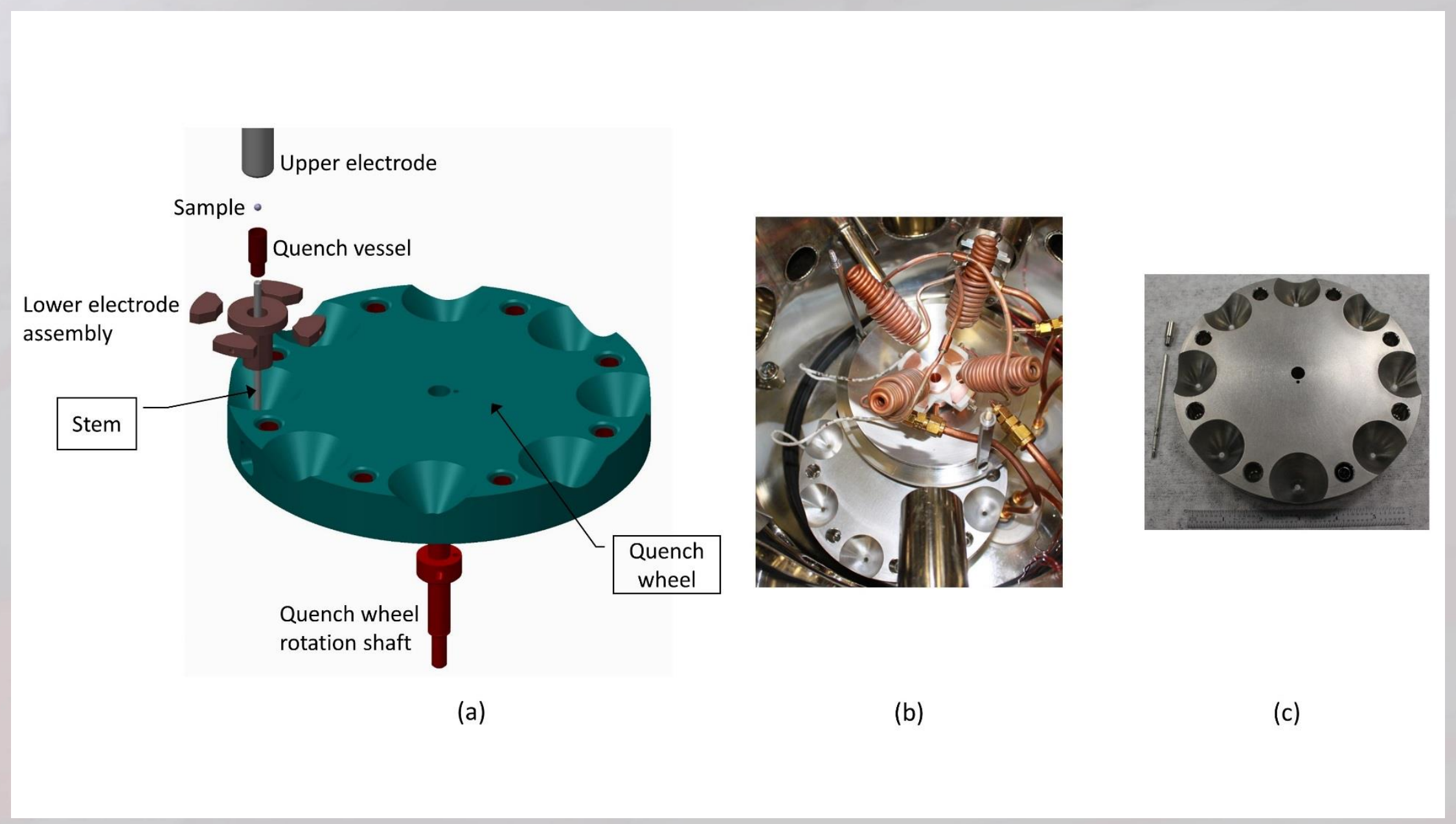




\section{Motivation}

- To preserve transient microstructures for quantitative metallographic analysis

- To freeze-in metastable phases for solidification path determination

- To rapidly solidify reactive melts while minimizing internal fluid flow

- To reduce fragmentation of structures associated with splat quench techniques

- To eliminate coarsening of microstructures to define assolidified dendrite shape

- To reduce both solid and liquid diffusion processes to observe partitioning in-situ

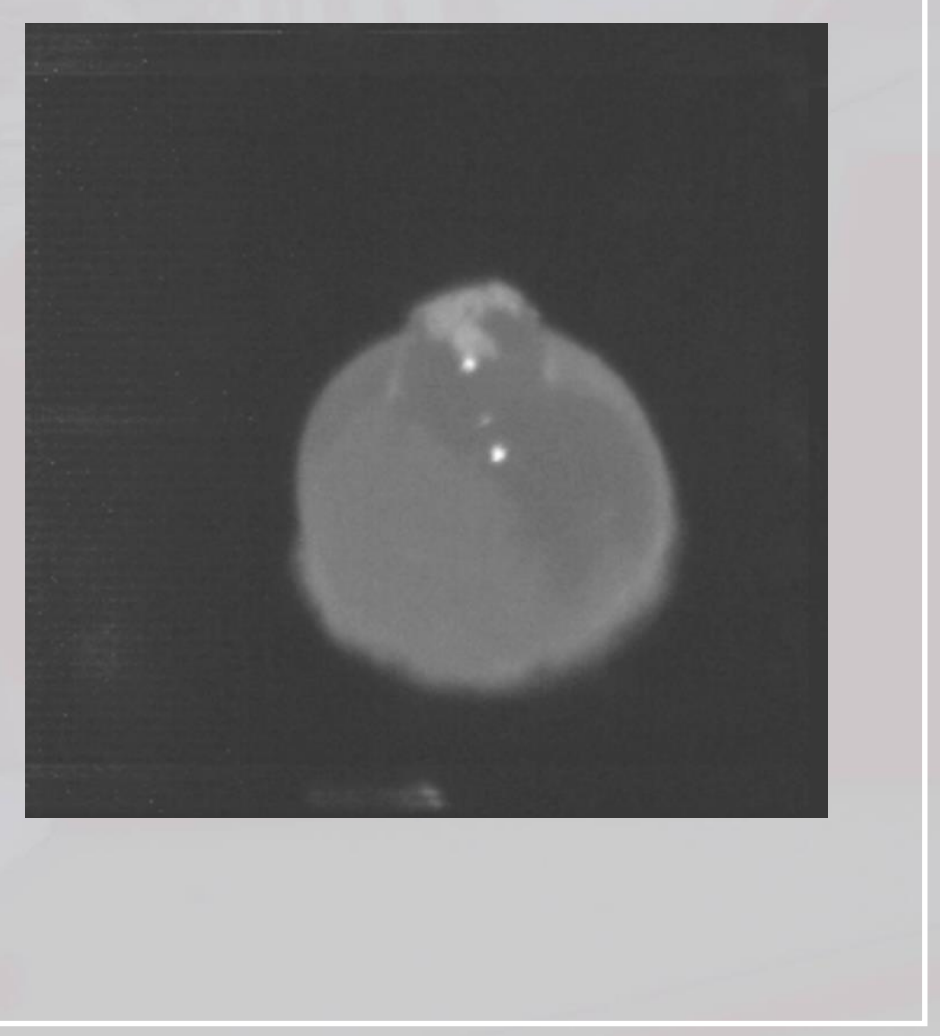




\section{Quench Medium}

- Indalloy 46L

- 61.0Ga - 25.0In - 13.0Sn - 1.0Zn

- Liquidus $=7.6 \mathrm{C}$

- Thermal Conductivity $=\sim 15 \mathrm{~W} / \mathrm{mK}$ (estimated by manufacturer)

- Gallium-Indium alloys have been proposed for similar studies by Koseki and Flemings

- T. Koseki and M.C. Flemings, "Solidification of Undercooled FeCr-Ni Alloys III: Phase Selection in Chilling", Metallurgical and Materials Transactions A, 28A (11) (1997), 2385-2395.

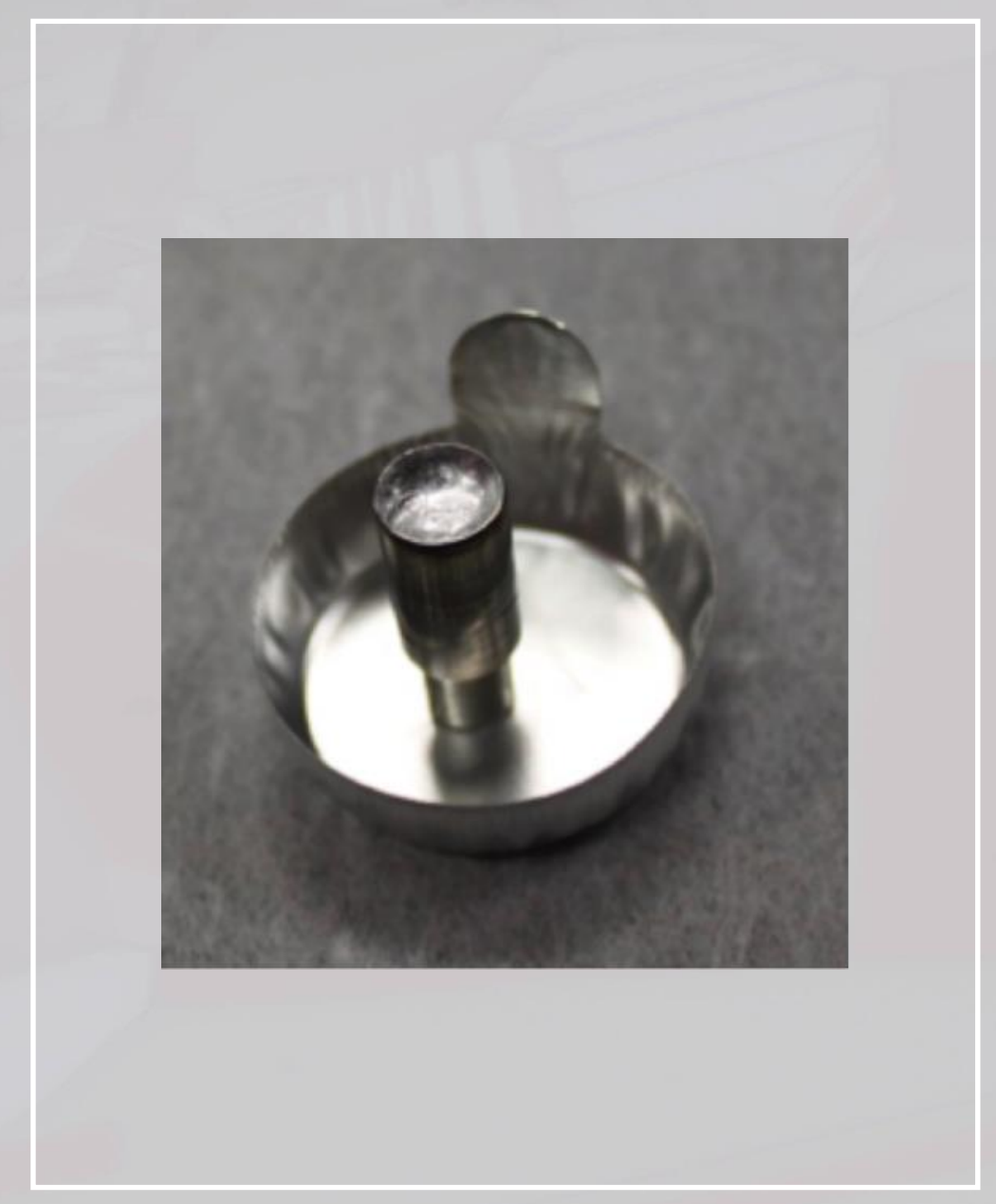




\section{Quench Sequence}

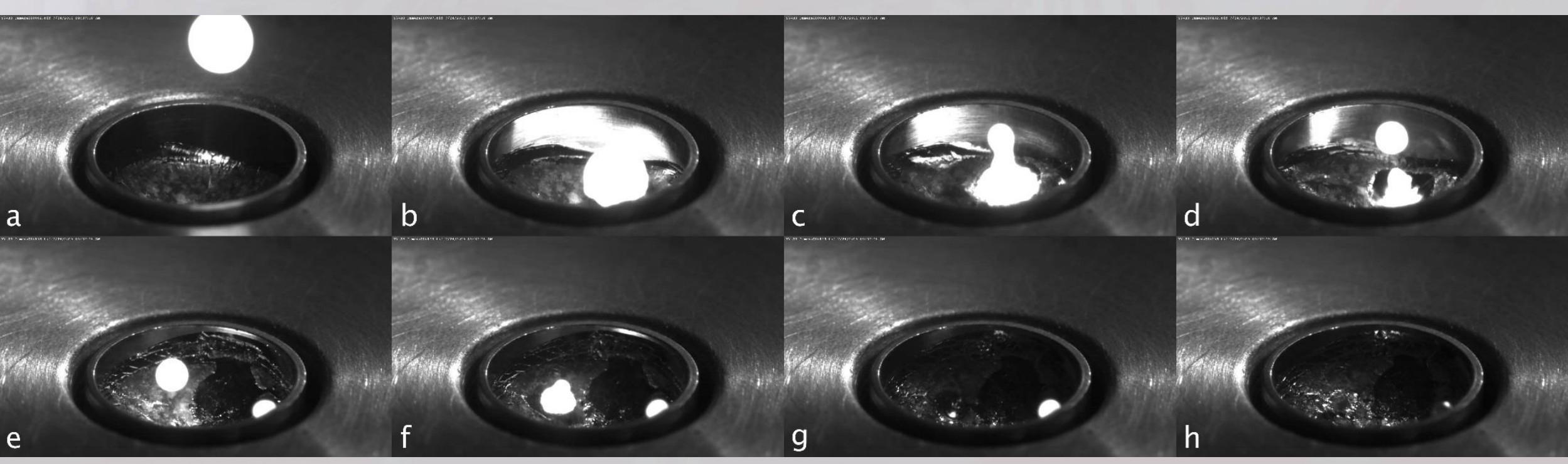




\section{Video}

\section{TIMS2016}




\section{Quench Sequence - Si58Co42}

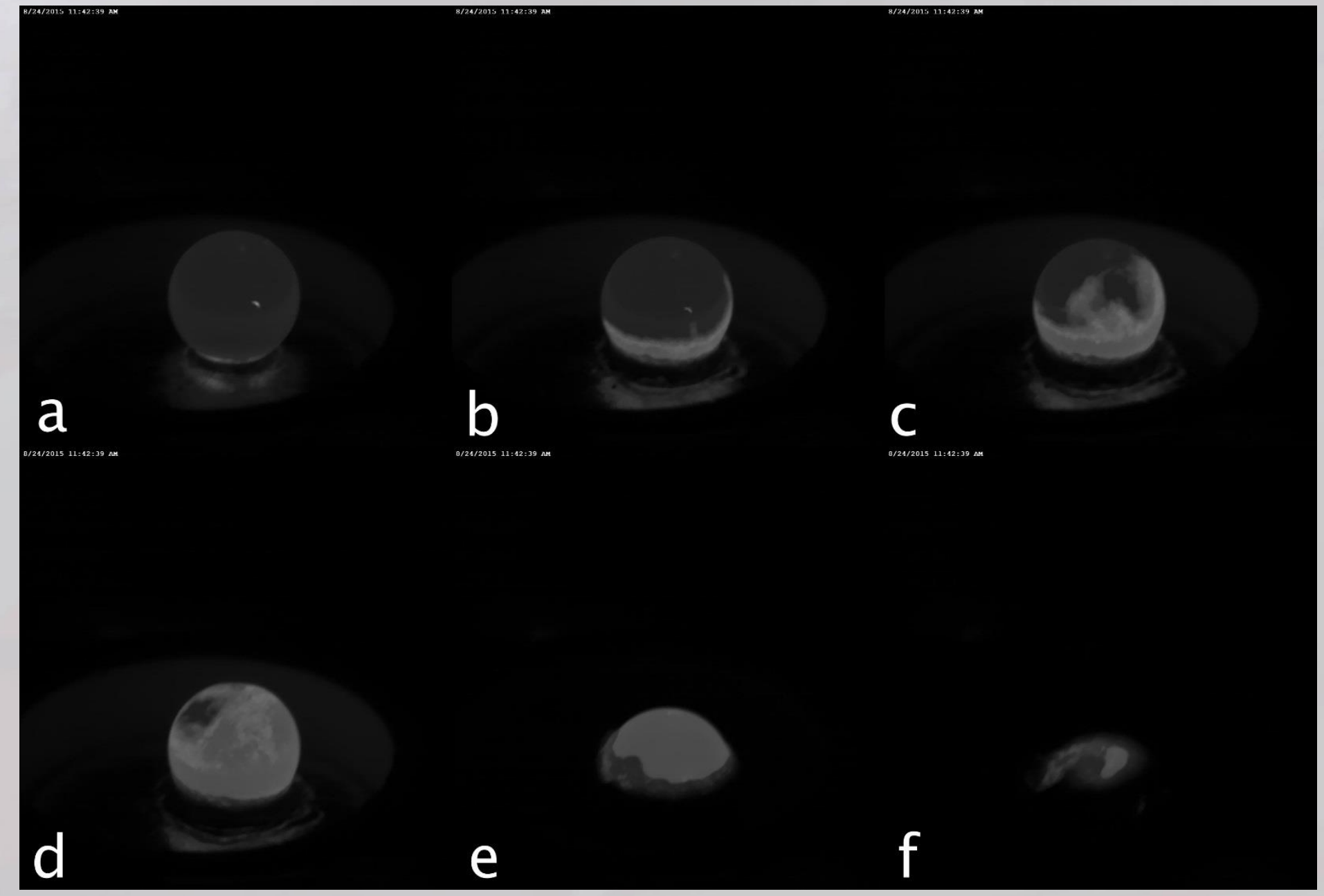




\section{Video of Si58Co42 Quench}




\section{Future Work}

- Eliminate surface dross

- Improve tracking of surface features to locate impact point/fluid closure point

- Calibrate quench rate as a function of depth below sample surface

- Optimize quench fluid removal from sample surfaces post-test

- Improve timing of droplet release from levitation field to minimize flight time 